\title{
A Comparison of Staging Systems for Squamous Cell Carcinoma of the Vulva
}

\author{
Michael P. Hopkins, M.D., ${ }^{1}$ Gary C. Reid, M.D., Caroline M. Johnston, M.D., \\ and George W. Morley, M.D. \\ Department of Obstetrics and Gynecology, University of Michigan Medical Center, Ann Arbor, Michigan
}

Received February 27, 1992

\begin{abstract}
A review of 172 patients with squamous cell cancer of the vulva treated at the University of Michigan Medical Center from 19751989 was performed to compare the 1988 FIGO Staging System to the 1970 FIGO Staging System. The stage distribution according to the 1970 FIGO Staging System was stage I, 65; stage II, 44; stage III, 50; and stage IV, 13. The cumulative 5-year survival under the old system was stage I, 94\%; stage II, 91\%; stage III, 36\%; and stage IV, 26\%. The distribution changed under the 1988 FIGO system to stage I, 58; stage II, 36; stage III, 49; stage IVA, 16; and stage IVB, 13. The cumulative survival also changed to stage I, 94\%; stage II, $89 \%$; stage III, $71 \%$; stage IVA, 19\%; and stage IVB, 8\%. The new FIGO stage distribution shifted for the worse due to the influence of positive lymph nodes found at the time of surgery. The survival was then analyzed for death from all causes. This was markedly decreased when compared to the cumulative corrected survival. This relates to the high number of other primary malignancies and the age of the patients. Among these 172 patients, other primary malignancies included squamous cell cancer of the cervix (11), squamous cell cancer of the vagina (2), endometrial cancer (3), squamous cell cancer of the lung (2), colon cancer (3), and others (6). An additional 5 patients died from myocardial infarction within 2 years of diagnosis. The new 1988 FIGO Staging System provides for better discrimination of survival between stages than the $\mathbf{1 9 7 0}$ FIGO Staging System. (c) 1992 Academic Press, Inc.
\end{abstract}

\section{INTRODUCTION}

Squamous cell carcinoma of the vulva remains localized for extended periods of time and spreads in an orderly fashion. Various risk factors have been evaluated for this disease and most authors agree that the lymph node status appears to be a critical determinant of survival. Prior to

Presented at the 23rd Annual Meeting of the Society of Gynecologic Oncologists, San Antonio, TX, March 15-18, 1992.

${ }^{1}$ To whom reprint requests should be addressed at Department of Obstetrics \& Gynecology, Northeastern Ohio Universities College of Medicine, 400 Wabash Avenue-ACC, Akron, OH 44307.
1988 the staging of vulvar cancer was based on the examiner's clinical evaluation of the lymph node status and the tumor location [1]. Thus, enlarged lymph nodes which were clinically suspicious or clinically positive influenced the stage regardless of the actual disease status. Alternatively, when lymph nodes were clinically negative, if they were found to be positive at the time of groin node dissection it would not change the final stage. Freidrich and DiPauloa in 1977 suggested that the staging system be changed to incorporate this information [2]. Over the last 20 years, radical vulvectomy, bilateral groin node disscction, with or without pelvic lymph node disscction, has become the standard therapy for this disease. Thus, most paticnts undcrgo surgery and the lymph node status is known. The FIGO staging system, therefore, was recently changed to a surgical staging system which accounts for the actual pathologic status of the lymph nodes [3]. The other change incorporated into the new staging system was the elimination of perineal involvement which previously classificd the patient as stage III.

This study was undertaken to compare the new surgical staging system to the old clinical staging system and dctermine if this new staging system provided a better predictor of survival between stages. An analysis was also done to evaluate the probability of dying from all causes during this time period.

\section{MATERIALS AND METHODS}

A review of all patients with squamous cell carcinoma of the vulva treated at the University of Michigan during the time period 1975-1989 was undertaken. All records were reviewed including the clinical charts, pathology reports, operative notes, and gynecologic tumor conference notes. All patients during this time period had been staged according to the 1970 FIGO TNM System. A separate review of these data was then done to stage patients 
TABLE 1

Changes in FIGO Stage when Comparing the 1970 Distribution by Stage to the 1988 Staging System

\begin{tabular}{|c|c|c|c|c|c|c|}
\hline \multirow{2}{*}{\multicolumn{2}{|c|}{ FIGO 1970}} & \multicolumn{5}{|c|}{ FIGO 1988} \\
\hline & & \multirow{2}{*}{$\begin{array}{c}\text { Stage } \\
\text { I }\end{array}$} & \multirow{2}{*}{$\begin{array}{c}\text { Stage } \\
\text { II }\end{array}$} & \multirow{2}{*}{$\begin{array}{c}\text { Stage } \\
\text { III }\end{array}$} & \multirow{2}{*}{$\begin{array}{l}\text { Stage } \\
\text { IVA }\end{array}$} & \multirow{2}{*}{$\begin{array}{l}\text { Stage } \\
\text { IVB }\end{array}$} \\
\hline Stage & No. & & & & & \\
\hline Stage I & 65 & 58 & - & 7 & - & - \\
\hline Stage II & 44 & - & 34 & 10 & - & - \\
\hline Stage III & 50 & - & 2 & 30 & 11 & 7 \\
\hline Stage IV & 13 & - & - & 2 & 5 & 6 \\
\hline Total & & 58 & 36 & 49 & 16 & 13 \\
\hline
\end{tabular}

according to the 1988 FIGO Staging System. Survival analysis was performed using a Kaplan-Meier Analysis [4]. The cumulative survival analyzes the probability of dying only from cancer, deleting patients as censored data when they die from other causes. The absolute survival analysis includes death from all causes. Statistical analysis was performed by the Northeastern Ohio University's College of Medicine, Biostatistics Department. A detailed analysis of separate risk factors which influenced survival utilizing the 1970 FIGO Staging System has been previously reported [5].

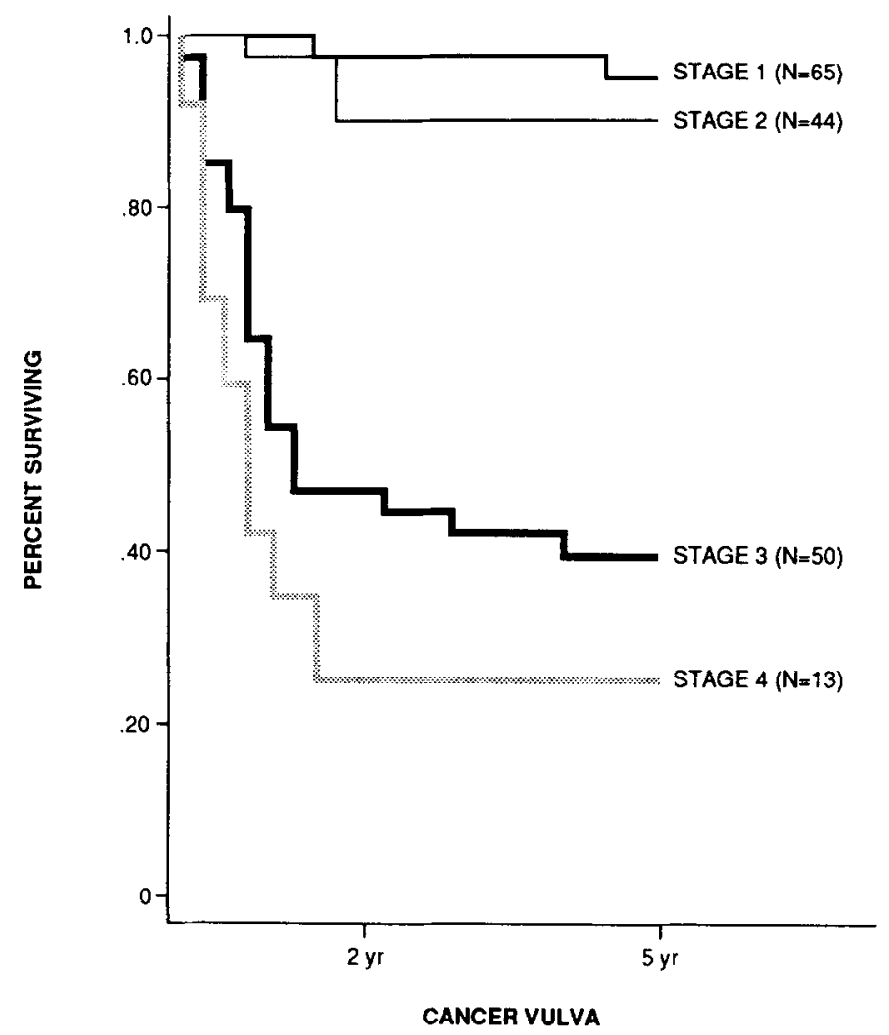

FIG. 1. Survival according to stage of disease utilizing the 1970 FIGO Clinical Staging System.

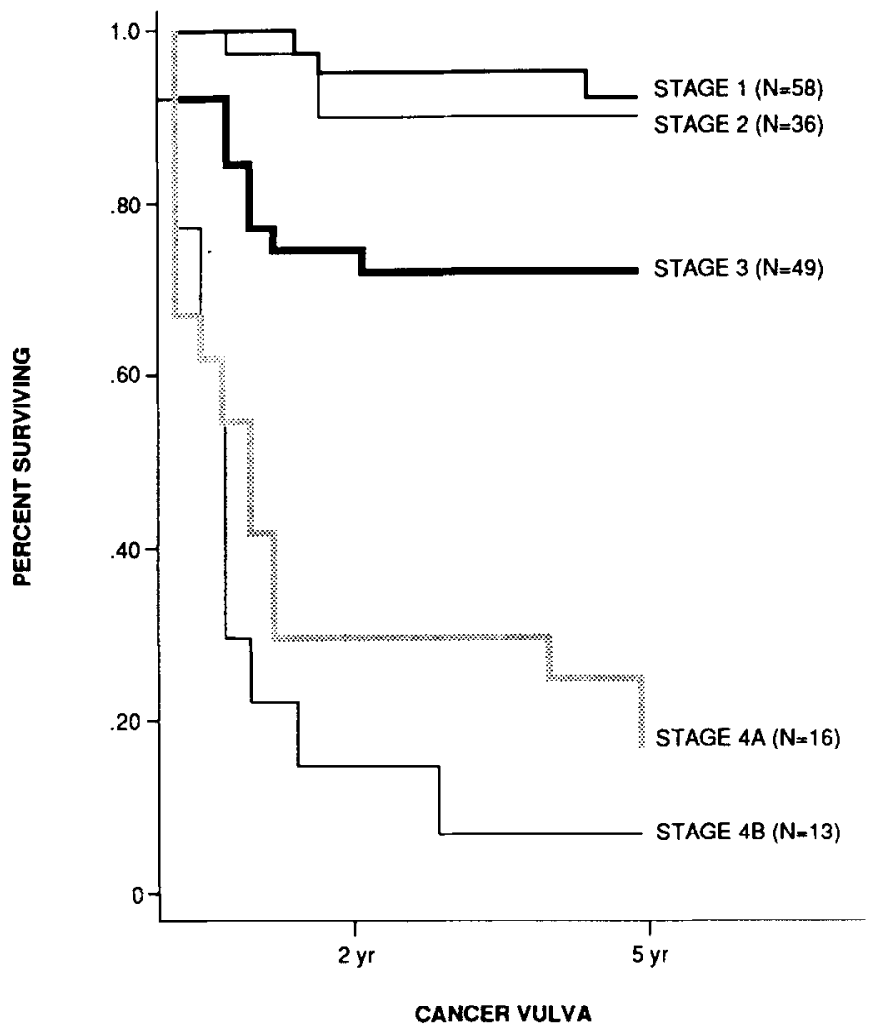

FIG. 2. Survival according to stage of disease utilizing the 1988 FIGO Surgical Staging System.

\section{RESULTS}

A total of 172 patients were treated for squamous cell carcinoma of the vulva during this time period. The distribution by the 1970 FIGO Staging System is compared to the 1988 FIGO Staging System in Table 1. A total of 7 patients with stage I disease were changed to stage III disease. These patients were staged higher under the new system due to the presence of positive unilateral lymph nodes. Ten patients with stage II disease were changed to Stage III under the new system. Again, all due to positive lymph nodes. There were no patients with stage I or II disease who changed their stage to IVA or IVB. The greatest change occurred in stage III disease where 50 patients were stage III in the 1970 system and only 30 of these 50 remained as stage III in the new system. Upstaging occurred for 18 patients, 11 to stage IVA and 7 to stage IVB. The 7 upstaged to IVB were all due to positive pelvic nodes. Two patients were downstaged to stage II disease. One patient had clinically positive nodes which on pathologic examination were negative. The other patient had a perineal lesion. Among the 13 patients with old FIGO stage IV disease, 2 were downstaged to stage III and the other 11 were split evenly between stage IVA and IVB.

The cumulative corrected 5-year survival for all patients was $71 \%$ with a cumulative 2 -year survival of $75 \%$. Survival curves constructed for the 1970 FIGO system and 


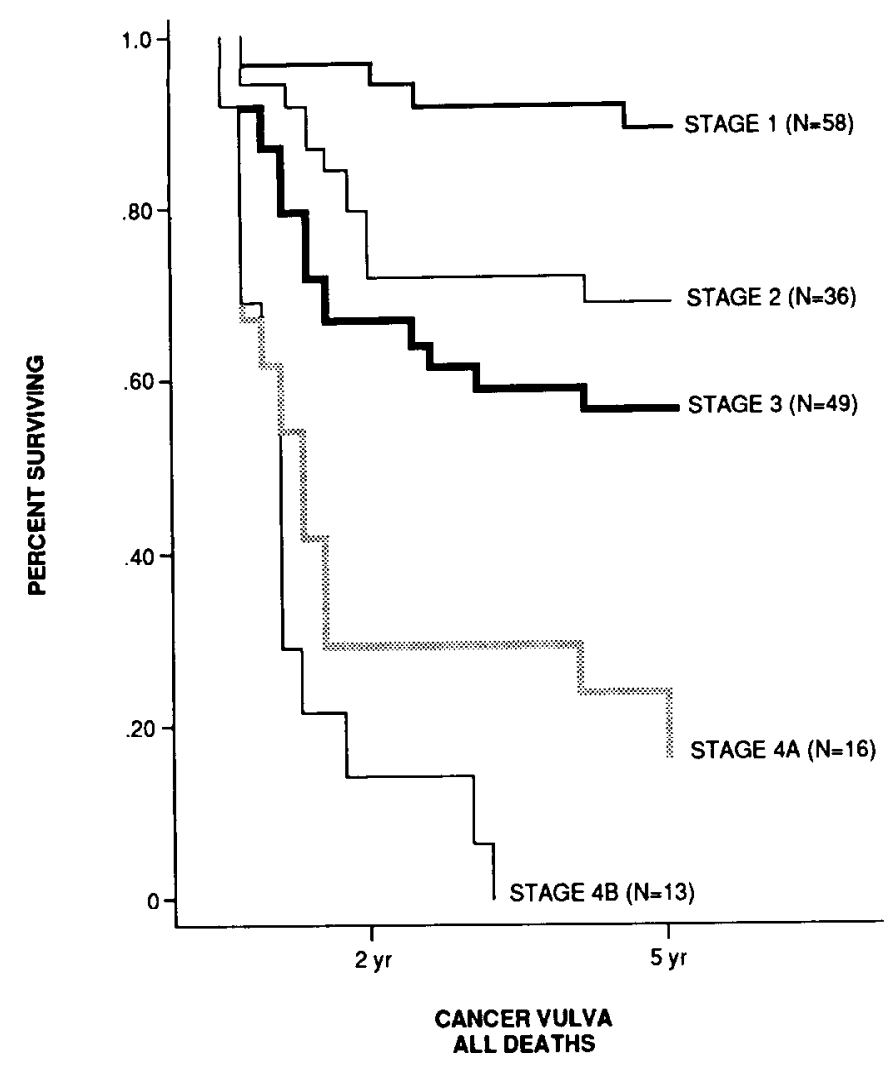

FIG. 3. Survival when accounting for death from all causes according to 1988 FIGO stage.

the 1988 FIGO system are presented in Figs. 1 and 2. Among patients with stages I and II disease, the cumulative 5-year survival changed very little. The 5-year survival in stage I remained the same at $94 \%$ while the cumulative 5-year survival for stage II changed from 91 to $89 \%$ in the new FIGO system. The greatest change occurred among patients with stage III disease. Under the old FIGO staging system in which stage III could include patients with pathologically proven bilateral positive groin nodes and positive pelvic nodes, the cumulative 5 -year survival was $36 \%$. Under the new system, where only patients with unilateral positive groin nodes are included, the 5-year survival was $71 \%$. Stage IV disease is now substaged into stages IVA, and IVB. Utilizing the new system there is a difference in cumulative survival of $19 \%$ for stage IVA versus $8 \%$ for stage IVB. As expected, the difference in survival between all the stages is significant $(P<0.0001)$.

A separate absolute survival analysis was performed where all causes of death were included. The absolute 5year survival was markedly decreased when compared to the cumulative corrected survival which only accounted for patients who died from vulvar cancer. The absolute 5 -year survival for all patients was only $62 \%$. Patients with stage I disease had an absolute survival of $90 \%$ and this dropped to 19 and $0 \%$ for stages IVA and IVB, respectively (Fig. 3). The lower absolute survival was influenced by the older age of the patients, other primary malignancies, and cardiac disease. The mean age of the patient population was 66 years with a range of 21 to 101 years. Second primary malignancies were frequent and the sites are listed in Table 2. Eight of these patients died from cancer other than vulvar cancer. Myocardial infarction within 2 years of the time of diagnosis was the cause of death in five patients.

\section{DISCUSSION}

The 1988 FIGO Staging System attempts to incorporate the known prognostic variables for squamous cell carcinoma of the vulva. The greatest change in staging has been from a clinical to a pathologic system including the lymph node status. The new staging system utilizes the known prognostic variables of positive unilateral (stage III) versus positive bilateral groin lymph nodes (stage IVA), versus positive pelvic lymph nodes (stage IVB). Under the old FIGO system there is little survival difference between patients with stage I or II disease and between patients with stage III or IV disease. When utilizing the new system there still remains little difference in survival between stages I and II. This is probably due to a number of factors. Under the old system, lymph nodes which were clinically negative but pathologically positive usually contained only microscopic disease and thus were less likely to significantly influence the survival. Under the new system, the only discrimination between stage I and stage II disease is the size of the primary tumor, less than $2 \mathrm{~cm}$ or greater than $2 \mathrm{~cm}$. This difference in size alone does not provide a great difference in survival.

In the old FIGO system there is a marked drop in survival for stages III and IV disease. Under the new FIGO system there are more pronounced survival differences between stages III, IVA, and IVB. Stage III disease carries a much better prognosis in the new system and this is almost certainly related to the presence of bilateral positive groin nodes now being classified as stage IVA disease. These results are similar to those reported

TABLE 2

Other Malignancies in Patients with Vulvar Cancer

\begin{tabular}{lr}
\hline Cervix invasive & 9 \\
Colon & 3 \\
Endometrium & 3 \\
Other invasive cancers & 4 \\
Breast & 2 \\
Lung & 2 \\
Vagina & 2 \\
Total & 25 \\
\hline
\end{tabular}


by Homesley where survival was $74 \%$ for stage III utilizing the new staging system and decreased to $31 \%$ for all patients with stage IV disease [6].

Because there is a difference in survival of $50 \%$ between stages III and IVA, other known prognostic risk factors will still be needed to predict which patients will survive. One predictive feature that is lost in the new staging system is the clinical impression of lymph node status. Iversen reported 100 patients with positive nodes. Those with clinically negative nodes had a $56 \%$ survival compared to $34 \%$ when nodes were clinically positive [7].

The cumulative disease-free survival is a method of analyzing treatment results and reporting only those patients who die from tumor-related deaths. Patients who die from other causes are removed as censored data but not as a death from disease. This provides an accurate survival picture as it relates to the cancer treatment but may not reflect the actual survival of the patient population. Because this patient population is elderly, the absolute survival provides an indicator of how many are alive regardless of the cause of death. It appears that in vulvar cancer the actual or absolute survival is markedly different from the cumulative disease-free survival. In our report a number of patients died from other causes. The population is elderly and almost $3 \%$ died from myocardial infarction. There also was a large number of secondary malignancies. Importantly, there was a high number of other squamous cell malignancies, especially involving the cervix, the vagina, and the lung. This finding concurs with the hypothesis of the field effect of a carcinogen involving the cervix, vagina, and vulva.

The new FIGO Staging System appears to be a better discriminator in determining survival from disease. The absolute survival appears to be markedly decreased when compared to the cumulative disease-free survival. The treatment for vulvar cancer includes radical surgery which often can be debilitating; therefore, a vigilant search should be done for other malignancies which may influence the course of treatment.

\section{REFERENCES}

1. Classification and staging of malignant tumours in the female pelvis, Acta Obstet. Gynecol. Scand. 50, 1-7 (1971).

2. Friedrich, E. G., and DiPaola, G. R. Postoperative staging of vulvar carcinoma: A retrospective study, Int. J. Gynecol. Obstet. 15, 270274 (1977).

3. Creasman, W. T. New gynecologic cancer staging, Obstet. Gynecol. 75, 287-288 (1990).

4. Kaplan, E. L., and Meier, P. Nonparametric estimation from incomplete observations, J. Am. Stat. Assoc. 53, 457-481 (1958).

5. Hopkins, M. P., Reid, G. C., Vettrano, I., and Morley, G. W. Squamous cell carcinoma of the vulva: Prognostic factors influencing survival, Gynecol. Oncol. 43, 113-117 (1991).

6. Homesley, H. D., Bundy, B. N., Sedlis, A., Yordan, E., Berek, J. S., Jahshan, A., and Mortel, R. Assessment of current International Federation of Gynecology and Obstetrics staging of vulvar carcinoma relative to prognostic factors for survival (a Gynecologic Oncology Group study), Am. J. Obstet. Gynecol. 164, 997-1004 (1991).

7. Iversen, $T$. The value of groin palpation in epidermoid carcinoma of the vulva, Gynecol. Oncol. 12, 291-295 (1981). 